\title{
Differences in food consumption under intermittent and continuous reinforcement schedules of water delivery: Some implications for schedule-induced behavior
}

\author{
CORA LEE WETHERINGTON \\ University of North Carolina, Charlotte, North Carolina \\ and \\ ANTHONY L. RILEY \\ The American University, Washington, D.C.
}

\begin{abstract}
Consumption of food pellets was examined in four water-deprived rats during 1 - $\mathrm{h}$ sessions in which water was presented once every 30,60 , or 120 sec independently of the rats' behavior according to three fixed-time (FT) schedules. Correlated with each FT condition was a continuous reinforcement (CRF) control condition in which the rats received, at the start of the session, the number of dipper presentations that were programmed to occur during the corresponding FT condition. During both the FT and CRF conditions, pellets per dipper presentation decreased and food intake rate increased with rate of water presentation, and there was a direct linear relation between $\log$ food intake and log water intake. For each of these three measures there was less eating under the FT condition than under the CRF condition, but the difference between the FT and CRF functions decreased at shorter FT values. These data are discussed in terms of the effects of amount of water on food consumption and the principle of temporal summation.
\end{abstract}

A large body of research has shown that when fooddeprived animals are intermittently presented small portions of food, they consume an excessive amount of water. This phenomenon is called "schedule-induced polydipsia" (SIP) (see Falk, 1969, 1971; Staddon, 1977; Wetherington, 1982). In addition to excessive fluid consumption, intermittent food has been reported to induce other excessive behaviors, including aggression, pica, escape, and wood-chewing (see Falk, 1971; Roper \& Crossland, 1982). Falk (1971) has argued that collectively these excessive behaviors, which occur under an intermittent schedule, form a class of behavior which he has termed "schedule-induced" or "adjunctive."

Given that intermittent food readily induces water consumption, the question of whether scheduling intermittent water produces excessive food consumption naturally arises. After several researchers reported failure to produce schedule-induction of eating by intermittent water (Carlisle, Shanab, \& Simpson, 1972; King, 1974; also see Allison \& Mack, 1982), Wetherington and Brown-

This research was supported in part by funds from the Foundation of the University of North Carolina at Charlotte and from the State of North Carolina. Manuscript preparation was supported by funds from National Science Foundation Grant BNS-8406445. This research was presented at the annual meeting of the Psychonomic Society in San Antonio, Texas, November 1984. Reprints may be obtained from Cora Lee Wetherington, Department of Psychology, University of North Carolina at Charlotte, Charlotte, NC 28223. stein (1979) raised the possibility that eating nevertheless might be under the control of intermittent water in terms of temporal patterning. They varied the interwater interval from 30 to $240 \mathrm{sec}$ and found that eating was indeed temporally modulated by the water schedule; this temporal modulation is a characteristic property of SIP. They also reported two other parallels with SIP: food ingestion rate increased and food responses per water presentation decreased with rate of water presentation.

There are at least two possible explanations for these three parallels between eating under spaced water and SIP. First, it is possible that eating under spaced water is, in fact, schedule induced. Prior reported failures at producing schedule-induced eating had tested for excessiveness at only one schedule value (Carlisle et al., 1972; King, 1974). And since Wetherington and Brownstein (1979) did not explicitly test for excessiveness, it is possible that some of the schedule values they examined would have produced schedule-induced eating. Successful demonstration of schedule-induced eating would be particularly important in view of Roper's (1981) recent contention that SIP may be the only schedule-induced behavior. A second possibility is that the three parallels between eating under intermittent water schedules and drinking under intermittent food schedules reported by Wetherington and Brownstein (1979) do not in any way imply a similar mechanism or process, that is, schedule-induction. $\mathrm{Ob}$ viously, a behavior can be temporally modulated by a 
schedule of food without being schedule induced. Moreover, it is possible that the other two functional relationships reported by Wetherington and Brownstein (1979) may simply reflect the effect of amount of water on food intake. In their research, changes in the interwater interval also produced changes in the amount of water per session. Thus, it is possible that the functional relationships between rate of eating and rate of water presentation and between food responses per water presentation and rate of water presentation were both due to the molar variable, overall amount of water per session, rather than the more molecular variable, intermittency provided by the individual intervals. Control of behavior by such molar events is common in the behavior-analytic literature (see Hineline, 1985, for a recent review).

The present experiment examined these two possibilities. Food intake was examined under several intermittent water schedules. For each schedule a control condition was conducted in which continuous reinforcement access to water was provided until each rat produced an amount equal to that provided by the intermittent schedule. This control procedure served as a baseline against which to assess the occurrence of schedule-induced eating, and it provided a test of the possibility that changes in food intake associated with the value of the intermittent water schedule may simply reflect changes in the overall amount of water per session provided by the schedule.

\section{METHOD}

\section{Subjects}

Four male Dublin-Sprague-Dawley rats, approximately 6 months old, served as subjects. They had previously been used briefly in an undergraduate psychology laboratory course.

\section{Apparatus}

Four operant conditioning chambers (BRS/LVE Model 1310) equipped with a pellet dispenser, water dipper, and two retractable levers were used. White noise was used to mask extraneous sounds. Standard electromechanical programming and data recording devices were located in an adjacent room.

\section{Procedure}

Barpress training. The rats were reduced to $80 \%$ of their ad-lib weights via food deprivation and were shaped to press the left-hand lever; each barpress produced a 45-mg pellet of food. After two sessions, a steady response rate was established. Next, the rats were subjected to a 22 -h water-deprivation regimen while they were trained to press the right-hand bar; each barpress produced a .04$\mathrm{ml}$ dipper of water. Two training sessions were required to establish a steady response rate. During shaping of the left-hand barpress, the right-hand lever was retracted, and during shaping of the right-hand barpress, the left-hand lever was retracted.

Twenty-three-hour water deprivation. After the four sessions of barpress training, the rats were provided free access to food and water in the home cages for 9 days. Thereafter, they were maintained on a 23-h water-deprivation regimen whereby they received water for $1 \mathrm{~h}$ in the home cage. Food was continuously available. The rats were allowed to adjust to this regimen for 9 days before the start of the experiment proper.

Experiment proper. In three separate conditions, each rat was exposed to fixed time (FT) $30-\mathrm{sec}$, FT $60-\mathrm{sec}$, and FT $120-\mathrm{sec}$ schedules in which a water dipper was presented automatically at the end of the fixed-time period. The food lever was extended during the entire session, and each barpress produced a single food pellet. All sessions lasted $1 \mathrm{~h}$. Sessions for the four rats were conducted simultaneously, and the FT conditions remained in effect until each rat displayed little day-to-day variability in the total number of food responses and in the distribution of food responses within the interwater interval. The order of the FT conditions and the corresponding number of sessions (in parentheses) were as follows: FT 60 (61), FT 30 (43), FT 120 (82).

For each of the three FT conditions, a continuous reinforcement (CRF) condition was conducted in which water-lever presses produced the water dipper until the rat received the number of presentations that were scheduled during the prior FT condition. Then the water lever was retracted for the remainder of the 1-h session. The number of water presentations per session during the control sessions for the FT 30 , FT 60 , and FT 120 schedules, respectively, was 120,60 , and 30 . The food lever was extended during the entire session, and each barpress produced a single food pellet. Each CRF condition was conducted immediately following the corresponding FT condition and lasted for 10 sessions.

\section{RESULTS}

All data are reported as the means of the last 5 days of each condition. Figure 1 shows the relative number of barpress responses for food as a function of relative time in the interwater interval under each of the FT schedules. Since the rats ate the food pellets as quickly as they produced them, these curves accurately reflect the temporal distribution of eating during the interwater interval. In general, the probability of eating was lowest immediately after and before water presentation; the highest probability occurred during the first third to half of the interwater interval. During the FT 120 condition, there was very little temporal control for Rats A2 and A4. Under the CRF conditions, there are no similar temporal distribution functions, since food responses rarely occurred between water responses; instead, food responses usually occurred only after the specified water deliveries were presented.

The top panel of Figure 2 shows the mean number of food pellets per water dipper (i.e., mean number of pellet deliveries divided by the number of water presentations) as a function of rate of water presentation. The abscissa values of 30,60 , and 120 correspond, respectively, to the FT 120, FT 60, and FT 30 schedules. Although this measure was computed in the same manner for both the FT and CRF conditions, it does not reflect the same temporal relationship between drinking and eating. Under FT, this measure truly reflects interwater interval eating. Under CRF, however, there was rarely any interwater eating; instead, eating typically did not occur until all the specified water deliveries were presented. Thus, under CRF, this measure does not reflect mean number of pellets per interwater interval, but, rather, food consumption per amount of water. These curves are generally decreasing during both the FT and CRF sessions, and the CRF functions are higher.

The middle panel shows the rate of food-pellet delivery (i.e., mean number of pellets produced during the 1-h 


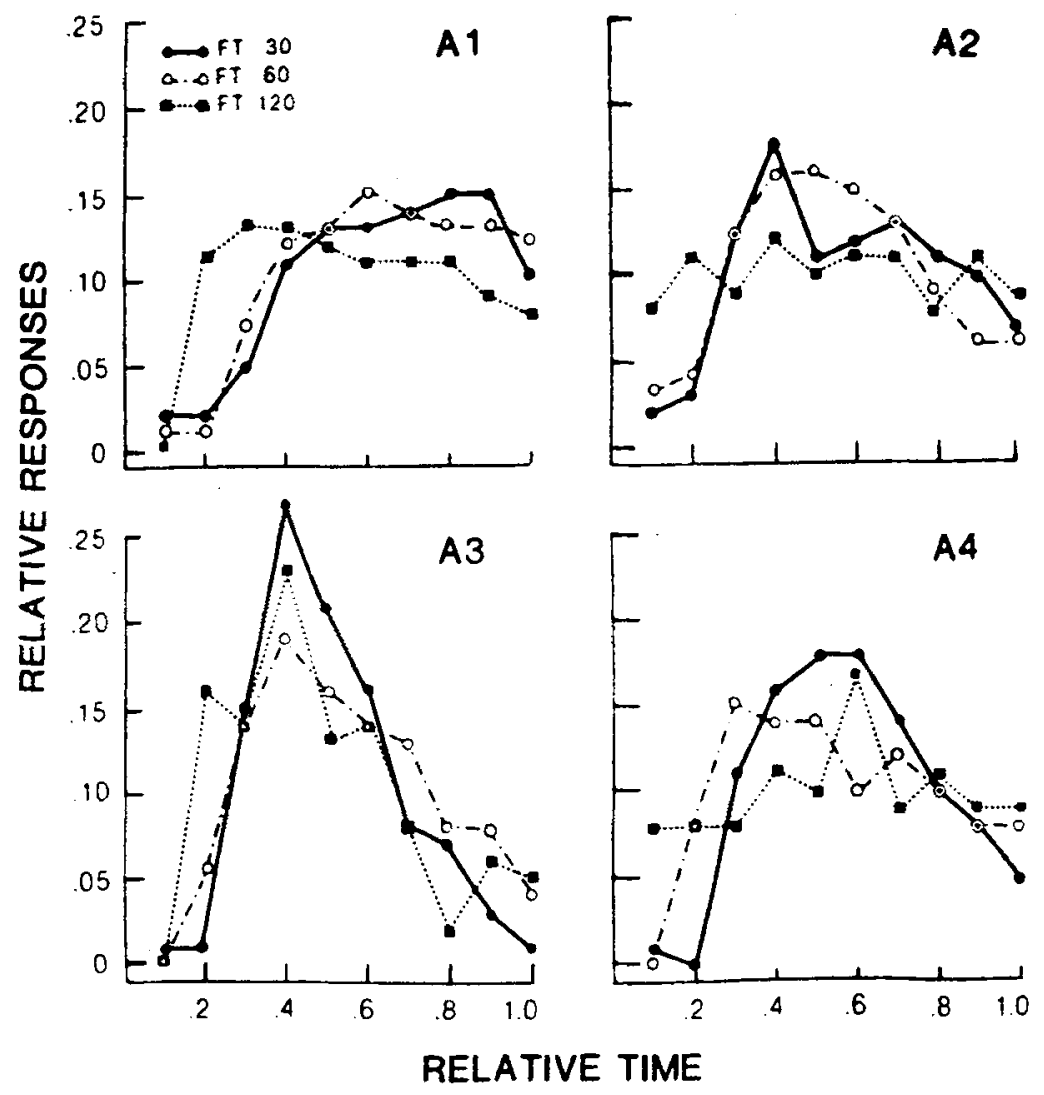

Figure 1. Relative number of food responses as a function of relative time in the interwater interval for each rat under each of the three FT schedules.

session divided by 60 ) as a function of rate of water presentation. During both the FT and CRF sessions, these curves are increasing, and again the CRF functions are higher.

Both measures of feeding in the top two panels of Figure 2 clearly indicate less feeding under the FT condition than under the corresponding CRF condition. To measure the difference in food intake under the FT and CRF schedules, the difference between the FT and CRF intakes was divided by the CRF intake, that is, (FT intake - CRF intake)/CRF intake. When the intake under FT and CRF are equal, the value of this measure is 0.00 , but as the difference between intake under FT and CRF increases, the value deviates from zero. Thus, this measure expresses the intake under FT relative to intake in the CRF condition. This measure is depicted in the bottom panel of Figure 2. It applies to both of the measures of feeding in the upper two panels, since all sessions were 1-h long and the sessions for a given FT-CRF pair contained an equal number of water presentations. The values ranged from -0.20 to -0.53 . Only in Rat A3's FT 60sec condition was there a failure to find less feeding under FT than under CRF. For all rats, the greatest difference between food intake under FT and CRF occurred at 30 water presentations per hour, that is, FT 120 . This difference diminished at higher rates of water presentation.
The relationship between the FT and CRF functions is further illustrated in Figure 3, which shows, in log-log coordinates, total food intake during the 1 -h sessions as a function of amount of water provided by the FT and CRF conditions. The abscissa values of $1.2,2.4$, and $4.8 \mathrm{ml}$ correspond to the FT 120 , FT 60 , and FT 30 conditions, respectively. The straight lines show the least squares best fit for the FT and CRF conditions. In both cases, a straight line provides a good fit to the data, as indicated by the high correlation coefficients shown for each curve. Note that the FT and CRF lines will intersect at large amounts of water, indicating a diminishing difference between intake under FT and CRF at higher amounts of water.

\section{DISCUSSION}

The data obtained under the FT conditions replicate three findings previously reported by Wetherington and Brownstein (1979): (1) Eating is temporally modulated by intermittent water presentations, a finding also reported by Myerson and Christiansen (1979); (2) pellets per dipper decrease with water rate; and (3) pellets per minute increase with water rate. The present research extends that of Wetherington and Brownstein (1979) by inclusion of CRF conditions. Comparison of the food-intake functions under FT and CRF (see Figures 2 and 3) yields three 

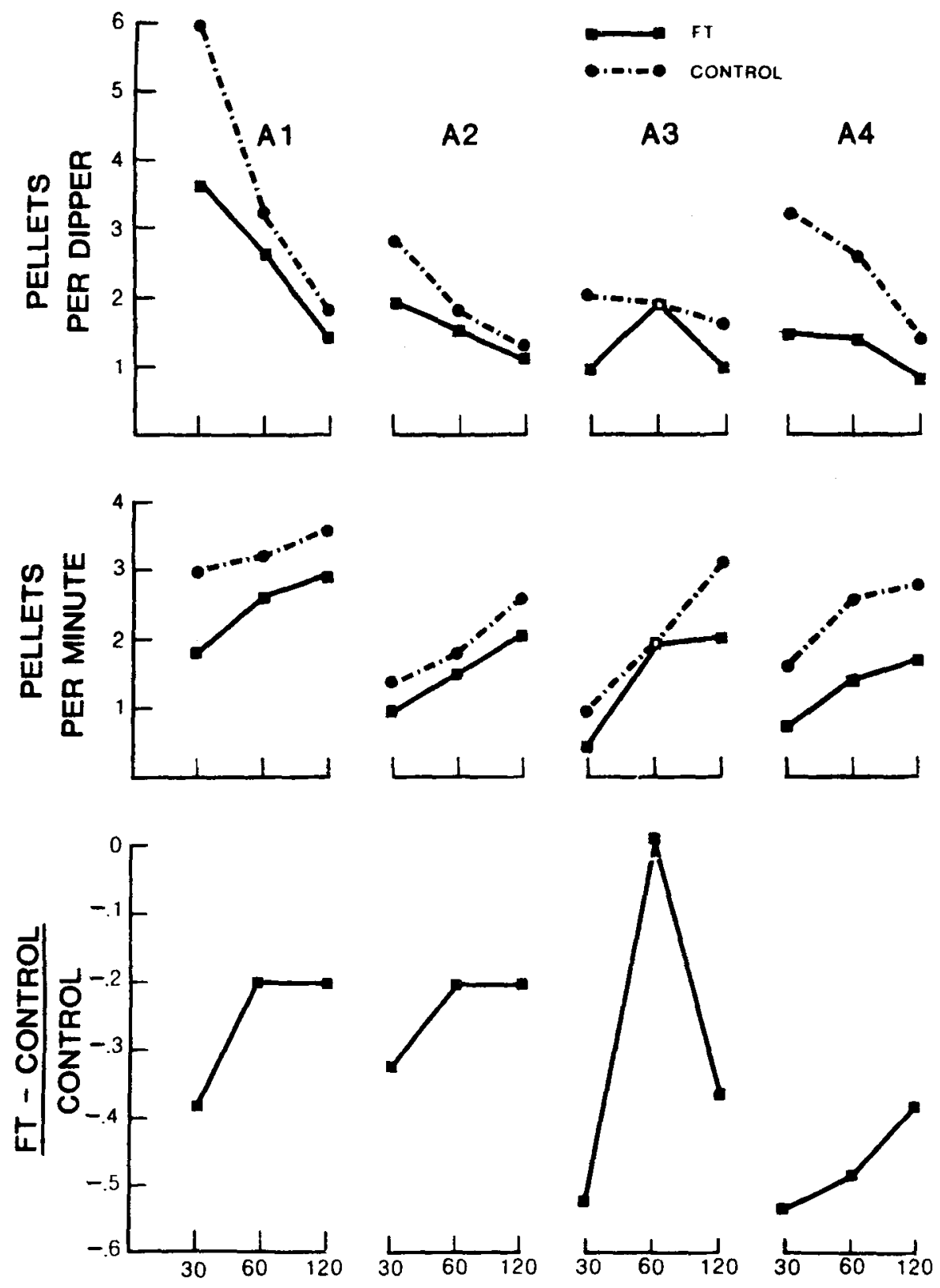

\section{WATER PRESENTATIONS PER HOUR}

Figure 2. Upper panel: Mean food pellets per dipper as a function of water presentations per hour during both the FT and control conditions for each of the four rats. Middle panel: Mean rate of food-pellet delivery as a function of rate of water presentation during both the FT and control conditions for each of the four rats. Lower panel: Difference between the food intake under FT and CRF divided by the intake under CRF. This measure applies to both of the measures in the upper and middle panels.

important findings: (1) The functions obtained under CRF were similar in shape to those obtained under FT, (2) the functions were clearly higher under CRF than under FT, and (3) the difference between the functions decreased at shorter FT values. These three comparisons provide a basis for determining (1) if schedule-induced eating occurred at any of the FT values, and (2) if the food intake changes accompanying changes in the FT value were due to amount of water per session rather than intermittency per se. We will discuss each of these questions in turn.

The finding that there was more food intake under CRF than under FT indicates that, under the range of schedule values tested, food intake was not schedule-induced. Thus, it appears that the similarity in functional relations observed between food intake under intermittent water schedules and polydipsic intake under intermittent food 


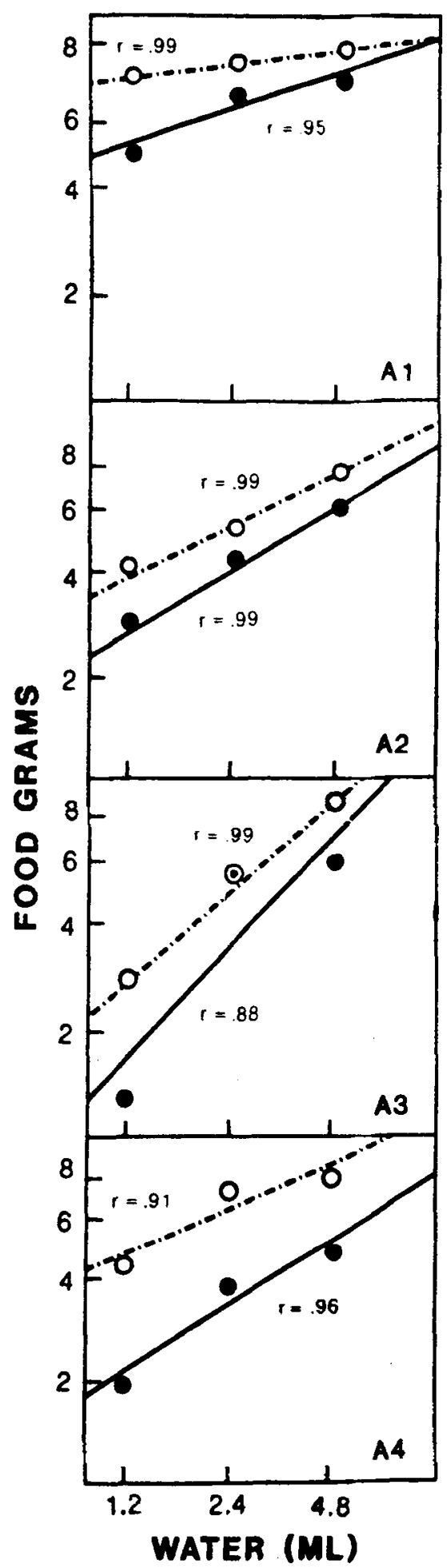

Figure 3. Food grams per session as a function of milliliters of water per session during the FT (closed circles) and CRF (open circles) conditions in log-log coordinates.

schedules is not due to a common schedule-induction mechanism. But, given that the difference between food intake under FT and CRF decreased with shorter FT schedules, it is possible that schedule-induced eating would have occurred at FT values shorter than $30 \mathrm{sec}$.
Perhaps so, but there is reason to suspect otherwise. As the FT value decreases, during a 1-h session, the number of water dipper deliveries per session increases. It seems reasonable to predict that as the number of water presentations per session increases, fewer water presentations would be consumed immediately upon delivery; instead, bouts of drinking would alternate with bouts of eating. Functionally, the FT water schedule would be converted to one in which drinking bouts occurred on a variabletime schedule. Similarly, as the value of the FT water schedule decreases, the number of water deliveries during the CRF control condition likewise increases. Again, it seems reasonable to predict that as the number of water deliveries provided in the CRF condition increases, eventually, bouts of drinking would begin to alternate with bouts of eating throughout the session and, like the FT schedule, the CRF schedule would be functionally converted to one in which drinking occurred on a variabletime schedule. In summary, this speculation suggests that as the FT value is shortened beyond $30 \mathrm{sec}$, the obtained pattern of drinking and eating under FT and CRF will approach equivalence and the amount of eating will likewise approach equivalence. Contrary to the typical view that CRF and FT schedules are qualitatively different, the present speculation suggests instead that they lie on a continuum.

The finding that the food-intake functions obtained under FT and CRF (see Figures 2 and 3) are of similar form certainly raises the possibility that the functions obtained under FT were due to the total amount of water per session rather than the intermittency provided by the individual interwater intervals. This possibility is supported by research (e.g., Allison \& Mack, 1982; Beck, 1964; Collier, 1964) which has explicitly examined various foodintake measures as a function of amount of water intake. Such research has shown that the pellets-per-dipper functions and the pellets-per-minute functions shown in Figure 2 and the linear relation between $\log$ food intake versus $\log$ water intake shown in Figure 3 are relationships commonly observed in rats exposed to various manipulations of amount of water. The FT and CRF functions observed in the present research, therefore, simply reflect basic relationships between food consumption and amount of water. Two important questions still remain unanswered: Why should more eating occur under a CRF schedule than under an FT schedule providing the same total amount of water? And why should this difference decrease with shorter FT values?

Answers may be provided by invoking the principle of temporal summation (Sherrington, 1906; Skinner, 1938). According to this principle, decreasing the time between stimulus presentations can be functionally equivalent to increasing the intensity or amount of the stimulus. Accordingly, when a fixed amount of water is given in rapid subunits, as in CRF, there is more temporal summation than when those subunits are spaced apart, as in FT. Thus, for any given FT-CRF pair, although the objective amount of water is the same, the CRF amount is functionally greater, hence producing more eating. This difference be- 
tween FT and CRF food intake would be expected to decrease with increases in the rate of water because as the FT decreases, the spacing of food during the FT session more closely approximates that of the CRF session. Eventually, an FT value should be reached which would correspond to the amount of water the rat would consume under a condition that provides for CRF access to water throughout the entire session. At this FT value, the difference between the level of eating under the FT and correlated CRF should approach equivalence.

The principle of temporal summation implies that food intake can be altered either by altering the amount of water or by holding amount constant and altering the time between water presentations. This is illustrated particularly well by Figure 3. Consider, for example, the CRF data point for Rat A2, which corresponds to $2.4 \mathrm{ml}$. Decreased food intake can be produced either by shifting to the associated FT schedule also providing $2.4 \mathrm{ml}$ (i.e., increasing the time between water presentations) or by shifting to the CRF schedule corresponding to $1.2 \mathrm{ml}$ of water (i.e., decreasing the amount of water). In this particular example, the CRF condition corresponding to $1.2 \mathrm{ml}$ produces the same food intake as the FT condition corresponding to $2.4 \mathrm{ml}$.

The present data have implications for the recent controversy over the definition of SIP and schedule-induced behavior in general (Cohen \& Looney, 1984; Roper, 1981; Timberlake, 1982; Wetherington \& Brownstein, 1982). Roper (1981) has argued that drinking, for example, should be called schedule-induced only if its occurrence is greater under an intermittent schedule than (1) under a CRF or massed food control (i.e., presentation en masse of the amount of food delivered under the intermittent schedule) and (2) in the absence of food. According to these criteria, the FT water presentations clearly did not induce feeding, since more food was consumed in the CRF conditions. Cohen and Looney (1984) recently argued against using the CRF or en masse controls as means by which to assess schedule induction and argued instead that only a no-food condition was necessary. In the present research, food intake during sessions containing no water was not examined. However, given the well-established finding that food consumption decreases in water-deprived rats and the finding in the present experiment that ingestion rate was directly related to water rate, it seems reasonable to suppose that had a no-water condition been run, very little food would have been consumed. If that were the case, then, according to Cohen and Looney's (1984) criterion, the present data, interestingly, would indicate that both the FT schedules and the CRF schedules generated schedule-induced eating.

Associated with the controversy regarding the definition of schedule induction is a concern over the best way to measure the strength or excessiveness of schedule-induced behavior. There have been two major ways in which SIP has been measured: water intake per pellet and ingestion rate. Based on the inverted- $U$-shaped function between water intake per pellet delivery and interpellet interval, polydipsic intake is often said to be most excessive at intermediate intervals. But, given that interpellet intervals of varying length provide varying opportunities to drink, some researchers have presented measurement of SIP as an ingestion rate, that is, water intake divided by total session time. In this case, polydipsic intake is maximal at short FT values (e.g., Staddon, 1977; Wetherington, 1979). In the present research, according to the first measure, that is, pellets per water presentation (Figure 1, upper panel), feeding was highest at 30 water presentations per hour (i.e., FT 120), whereas according to the second measure, that is, pellets per minute (Figure 1, middle panel), feeding was highest at 120 water presentations per hour (i.e., FT 30). Both of these measures, however, follow directly from the linear relationship between log food consumption and log water consumption (Figure 3). The slope of this relationship is less than 1.0, indicating that, although increasing amounts of water intake produce increasing amounts of food intake, the proportionate increase in food intake is less than the proportionate increase in water intake, thus resulting in a decrease in the ratio of food consumption per water consumption (i.e., pellets per dipper) with amount of water consumption. The direct relation between pellets per minute and rate of water presentation (Figure 2, middle panel) also follows directly from the $\log -\log$ relation in Figure 3; the values of the abscissa and ordinate of the latter data points simply reflect multiplication by the weight of the food pellets $(45 \mathrm{mg})$ and the size of the water dipper $(0.04 \mathrm{ml})$, respectively. Given that both the pellets-per-dipper function and the pellets-per-minute function follow directly from the linear relation between log food intake and log water intake, there is no basis for claiming that either measure is preferable.

The fact that there is disagreement over the best operational definition of schedule induction does not necessarily imply that there is, in fact, a best definition or that there is even a necessity for the term. The data upon which the term was originally based were simple: the noncontingent delivery of food generates a level of water intake which, by a number of arbitrary criteria described by Falk (1969), appeared excessive. Current controversy over defining schedule induction, then, is simply controversy over defining excessiveness. The major task for researchers, it seems, should be to account for the level of intake regardless of any arbitrary rules we might adopt in order to say that it is excessive. The present research suggests that the best route to understanding this level of water intake may be to examine drinking under a variety of parametric manipulations of food spacing, food rate, and food amount, and establish functional equivalences among them. This strategy would allow us to view apparent excessiveness simply as instances of certain values of fundamental functional relationships (see Catania, 1979). It would obviate notions of schedule induction (Cohen \& Looney, 1984; Roper, 1981; Timberlake, 1982; Wetherington \& Brownstein, 1982) and, instead, focus attention on seeking explanations of behavioral regularities rather than classifying them. 


\section{REFERENCES}

Allison, J., \& MACK, R. (1982). Polydipsia and autoshaping: Drinking and'leverpressing as substitutes for eating. Animal Learning \& Behavior, 10, 465-475.

BECK, R. C. (1964). Some effects of restricted water intake on consummatory behavior of the rat. In M. J. Wayner (Ed.), Thirst (pp. 305-316). New York: Pergamon Press.

Carlisle, H. J., Shanab, M. E., \& Simpson, C. W. (1972). Scheduleinduced behaviors: Effect of intermittent water reinforcement on food intake and body temperature. Psychonomic Science, 26, 35-36.

Catania, A. C. (1979). Learning. Englewood Cliffs, NJ: Prentice-Hall.

COHEN, P. S., \& LOONEY, T. A. (1984). Induction by reinforcer schedules. Journal of the Experimental Analysis of Behavior, 41, 345-353.

Colluer, G. (1964). Thirst as a determinant of reinforcement. In M. J. Wayner (Ed.), Thirst (pp. 287-303). New York: Pergamon Press.

FALK, J. L. (1969). Conditions producing psychogenic polydipsia in animals. Annals of the New York Academy of Sciences, 157, 569-593.

FALK, J. L. (1971). The nature and determinants of adjunctive behavior. Physiology \& Behavior, 6, 577-588.

HiNeline, P. N. (1985). Aversive control: A separate domain? Journal of the Experimental Analysis of Behavior, 42, 495-509.

KING, G. D. (1974). Wheel running in the rat induced by a fixed-time presentation of water. Animal Learning \& Behavior, 2, 325-328.

Myerson, J., \& Christiansen, B. (1979). Temporal control of eating on periodic water schedules. Physiology \& Behavior, 23, 279-282.
ROPER, T. J. (1981). What is meant by the term "schedule-induced," and how general is schedule induction? Animal Learning \& Behavior, 9, 433-440.

RoPER, T. J., \& Crossland, G. (1982). Schedule-induced wood-chewing in rats and its dependence on body weight. Animal Learning \& Behavior, 10, 65-71.

SherRington, C. S. (1906). The integrative action of the nervous system. New Haven, CT: Yale University.

SkINNER, B. F. (1938). The behavior of organisms. New York: Appleton-Century.

Staddon, J. E. R. (1977). Schedule-induced behavior. In W. K. Honig \& J. E. R. Staddon (Eds.), Handbook of operant behavior (pp. 125152). Englewood Cliffs, NJ: Prentice-Hall.

Timberlake, W. (1982). Controls and schedule-induced behavior. Animal Learning \& Behavior, 10, 535-536.

WETHERINGTON, C. L. (1979). Schedule-induced drinking: Rate of food delivery and Herrnstein's equation. Joumal of the Experimental Analysis of Behavior, 32, 323-333.

WETHERINGTON, C. L. (1982). Is adjunctive behavior a third class of behavior? Neuroscience \& Biobehavioral Reviews, 6, 329-350.

Wetherington, C. L., \& Brownstein, A. J. (1979). Schedule control of eating by fixed-time schedules of water presentation. Animal Learning \& Behavior, 7, 38-40.

Wetherington, C. L., \& Brownstein, A. J. (1982). Comments on Roper's discussion of the language and generality of schedule-induced behavior. Animal Learning \& Behavior, 10, 537-539.

(Manuscript received February 20, 1985;

revision accepted for publication July 22,1985 .) 